\title{
THERE AND BACK AGAIN: \\ Lessons Learned on the Way to the Market
}

\author{
Rance Cleaveland \\ Department of Computer Science, University of Maryland \& \\ Fraunhofer USA Center for Experimental Software Engineering \& \\ Reactive Systems Inc. \\ rance@cs. umd.edu
}

\begin{abstract}
In 1999 three formal-methods researchers, including the speaker, founded a company to commercialize formal modeling and verification technology for envisioned telecommunications customers. Eight years later, the company sells testing tools to embedded control software developers in the automotive, aerospace and related industries. This talk will describe the journey taken by the company during its evolution, why this journey was both less and more far than it seems, and how the speaker's views on the practical utility of mathematically oriented software research changed along the way.
\end{abstract}

